\title{
Article \\ Macroscopic Traffic-Flow Modelling Based on Gap-Filling Behavior of Heterogeneous Traffic
}

\author{
Muhammad Umair Khan ${ }^{1}$, Salman Saeed ${ }^{1}$, Moncef L. Nehdi ${ }^{2, *}$ (D) and Rashid Rehan ${ }^{1}$ (D) \\ 1 National Institute of Urban Infrastructure Planning, University of Engineering \& Technology, \\ Peshawar 25000, Pakistan; umaircivil1@gmail.com (M.U.K.); salmansaeed@uetpeshawar.edu.pk (S.S.); \\ rashid@uetpeshawar.edu.pk (R.R.) \\ 2 Department of Civil and Environmental Engineering, Western University, London, ON N6A 5B9, Canada \\ * Correspondence: mnehdi@uwo.ca; Tel.: +1-519-661-2111 (ext. 88308)
}

check for updates

Citation: Khan, M.U.; Saeed, S.; Nehdi, M.L.; Rehan, R. Macroscopic Traffic-Flow Modelling Based on GapFilling Behavior of Heterogeneous Traffic. Appl. Sci. 2021, 11, 4278. https://doi.org/10.3390/app1109 4278

Academic Editor: Luís Picado Santos

Received: 3 April 2021

Accepted: 5 May 2021

Published: 9 May 2021

Publisher's Note: MDPI stays neutral with regard to jurisdictional claims in published maps and institutional affiliations.

Copyright: (c) 2021 by the authors. Licensee MDPI, Basel, Switzerland. This article is an open access article distributed under the terms and conditions of the Creative Commons Attribution (CC BY) license (https:// creativecommons.org/licenses/by/ $4.0 /)$.

\begin{abstract}
Traffic-flow modelling has been of prime interest to traffic engineers and planners since the mid-20th century. Most traffic-flow models were developed for the purpose of characterizing homogeneous traffic flow. Some of these models are extended to characterize the complex interactions involved in heterogeneous traffic flow. Existing heterogeneous traffic-flow models do not characterize the driver behavior leading to gap filling in heterogeneous traffic conditions. This study aimed at explaining the gap-filling behavior in heterogeneous traffic flow by using the effusion model of gas particles. The driver's behavior leading to gap filling in heterogeneous traffic was characterized through developing analogies between the traffic flow and the Maxwell-Boltzmann equation for effusion of gases. This model was subsequently incorporated into the Payne-Whitham (PW) model by replacing the constant anticipation term. The proposed model was numerically approximated by using Roe's scheme, and numerical simulation of the proposed model was then carried out by using MATLAB. The results of the proposed and PW models were therefore compared. It is concluded that the new model proposed in this study not only produces better results compared to the PW model, but also better captures the expected reality. The main difference between the behavior of the two models is that the effect of bottleneck in the density of traffic is propagated in the form of a shockwave travelling backwards in time in the new model, while the PW model does not exhibit this effect.
\end{abstract}

Keywords: traffic-flow modelling; heterogeneous traffic; gap filling; driver behavior; effusion of gases

\section{Introduction}

While the recent rapid pace of urbanization has modernized the living standards, it has been associated with attendant problems such as traffic congestion and environmental pollution [1]. Population growth and increased vehicle ownership are a major reason of traffic congestion. The rapid increase in the number of vehicles on roads also contributes significantly to the emission of greenhouse gases. Urban traffic congestion leads to severe economic and environmental impacts, as well as the loss of human lives in traffic accidents [2]. In recent years, major advancements have been made in the automotive industry and concepts like Automated Cruise Control (ACC) and Highly Automated Driving (HAD) have been introduced, out of which a few have materialized, while others are still in the testing or introductory stages. Along with these developments, new concepts like Smart Roads have also been introduced. These types of roads are equipped with such devices that provide monitoring of road conditions, analysis of the driver's behavior, congestion warnings, route selection, etc. [3]. Another interesting concept is that of the Lane Support Systems (LSS), which aims at reducing potential crashes by alerting the drivers or autonomous vehicles when they are about to drift out of a lane. Such Smart Roads have their advantages and disadvantages [3], and LSS may bear good experimental performance in certain conditions, as indicated by Pappalardo et al. [4]. However, the 
practical implementation of these systems in the near future seems far-fetched in developed countries, let alone in the developing countries where heterogeneous traffic conditions are more prevalent. In addition, researchers are now focusing on providing traffic planners with tools helping them in controlling traffic facilities in order to mitigate traffic congestion. In this regard, a PDDL+ approach was introduced by Vallati et al. [5], which controls traffic lights to aid in congestion reduction. Latest research interests include developing models to characterize the vehicular travel times. Zambrano-Martinez et al. [6] developed an equation that characterizes travel times and then classified urban roads on the basis of those travel times. However, despite what the future may bring, traffic congestion and its consequences must be addressed presently. These issues may be mitigated by analyzing traffic flows and then proposing effective solutions. For the analysis of traffic flow, the first step is to capture the behavior of traffic using mathematical equations, which is called traffic-flow modelling.

Three approaches to traffic-flow modelling are usually adopted: microscopic, macroscopic, and mesoscopic modelling. Microscopic models, such as those in References $[7,8]$, deal with the behavior of individual vehicles. Each vehicle in the traffic stream is modelled by using a separate Ordinary Differential Equation (ODE). Conversely, macroscopic models [9-12] focus on the aggregate behavior of the traffic stream and involve three parameters of prime interest: traffic density (number of vehicles per unit length per lane), speed, and flow (number of vehicles crossing a point per unit time). Lastly, mesoscopic models, for example [13], are a combination of both microscopic and macroscopic models and, hence, act as a bridge between the two approaches. This research focuses on macroscopic modelling because of its relatively simpler computations.

Traffic flow is characterized either as homogeneous or heterogeneous flow. In homogeneous traffic flow, there is strict lane discipline with traffic mainly composed of motorized vehicles, the dimensions of which do not vary much from each other [14]. Conversely, in heterogeneous traffic flow, the presence of both motorized and non-motorized vehicles is accompanied by very loose or no lane discipline at all [15]. In such traffic conditions, the gap-filling behavior is prevalent in which the drivers tend to escape the traffic behind by occupying the gaps they see ahead of them. Because of this, different vehicle types follow different levels of service resulting in complex interactions between the vehicles and causing severe disturbances within the traffic stream [16].

The first macroscopic traffic-flow model was developed by Lighthill and Whitham [9] and then independently by Richards [10]. This model is referred to as the Lighthill-WithamRichards (LWR) model and is composed of a single equation based on the law of conservation of mass. The LWR model is relatively simple to implement, however, it assumes equilibrium velocity distributions at every instant. In equilibrium velocity distribution, velocity is a unique function of the traffic density. Consequently, the velocity of the vehicle is adjusted instantaneously, implying that the relaxation time is zero, which is not possible. The relaxation time is that required by a vehicle to achieve the desired speed after it encounters an obstruction. Thus, this model ignores the transitions in traffic flow [17]. Payne [11] and Whitham [12] proposed a two-equation model in which the first equation is the same as the LWR model, while the second equation characterizes the traffic acceleration. The PW model is represented as follows:

$$
\begin{gathered}
\frac{\partial \rho}{\partial t}+\frac{\partial(\rho v(\rho))}{\partial x}=0 \\
\frac{\partial v}{\partial t}+\frac{v \partial v}{\partial x}=-\frac{C_{0}^{2}}{\rho} \frac{\partial \rho}{\partial x}+\frac{v(\rho)-v}{\tau}
\end{gathered}
$$

where $C_{0}$ is called the driver's anticipation constant and $\tau$ is the relaxation time. While the PW model improves the LWR model, because of the constant $C_{0}$, it always assumes similar behavior for all the vehicles in the traffic stream [17]. Daganzo [18] criticized the PW model for resulting in negative velocities. He also discussed the flaw in treating the 
traffic flow like a fluid flow by arguing that in fluids, a particle's motion is affected by the particles from all the sides, while in the traffic flow, a vehicle's motion is mainly affected by the front vehicle. Daganzo also argued that vehicles have individual personalities (driver behavior), while molecules lack that feature. For similar reasons, Aw and Rascle [19] criticized relating traffic flow to fluid flow. They introduced a pressure term in their model, which is an increasing function of the traffic density. However, Aw and Rascle's model ignores the velocity profile of the traffic, so that traffic adjustments only depend on forward traffic density conditions [20]. Khan [20] improved the PW model by using the kinematic equation of motion to characterize the transitions via considering the spatial changes in traffic density. When simulated, this model produced better results than the PW model. However, at higher velocities, its application is limited because of the fact that vehicles adjust their velocities very quickly, thus resulting in unrealistic relaxation time [21]. Accordingly, Khan and Gulliver [21] presented an improved version of the previous model by modifying the relaxation term and introducing safe velocity and a regulation parameter which assumes different values for drivers having different attitudes. Detailed work has been done on improving the PW model, which can be studied further in the pertinent review by Mohan and Ramadurai [22].

The models discussed above are better suited to homogeneous traffic flow. They cannot be adequately used for heterogeneous traffic flow because they may not be able to characterize the complex interactions between vehicles and severe disturbances within the traffic stream due to the gap-filling behavior in heterogeneous traffic. Some of the models which have been extended to heterogeneous traffic conditions are discussed subsequently. For instance, Zhang and Jin [23] extended the first order LWR model to heterogeneous traffic conditions having two vehicular classes and developed it for free flow and congested flow regimes. Despite satisfactory results, the extended model has the limitations of ignoring slower vehicles as an obstruction to the faster vehicles. Additionally, it neither captures the overtaking of slower vehicles by faster vehicles, nor the driver behavior. Ngoduy [24] extended the first order LWR model for heterogeneous traffic by introducing randomness in the driver behavior through a stochastic setting. This model gave good results for heterogeneous traffic flow. However, it is only based on a single equation. Higher order models can better represent heterogeneous traffic conditions since they can also characterize the traffic acceleration, which is of prime interest in the case of heterogeneous traffic flow [24]. Gupta and Dhiman [25] used the concept of lateral headway to explain the lane changing behavior in heterogeneous traffic. Their model, however, fails to characterize the heterogeneity in driver behavior. Mohan and Ramadurai [26] developed a secondorder traffic-flow model for heterogeneous traffic. They incorporated the concept of area occupancy instead of traffic density. They also introduced traffic pressure as a function of the area occupancy. This model explains the bottleneck phenomenon better than the earlier models. However, it has the limitation of not characterizing the driver behavior in heterogeneous traffic conditions.

It becomes evident from the discussion above that conventional macroscopic trafficflow models cannot characterize heterogeneous traffic. Some of these models have been extended to heterogeneous traffic conditions, yet they are limited because of their inability to characterize the driver behavior that leads to the gap filling in heterogeneous traffic.

\section{Macroscopic Models Based on Fluid Flow}

Developing traffic-flow models using the fluid flow has become the norm in the field of traffic engineering. In addition to the LWR and PW models, several other researchers also developed traffic-flow models using the fluid-flow theory. Prigogine and Herman [27] were one of the earliest to derive a traffic-flow model by conceptualizing the kinetic theory of gases. In their model, vehicles are treated as gas particles, while vehicle interaction is taken as analogous to the collision between gas particles. This model is suitable for dilute traffic conditions only. Fontana [28] presented another traffic-flow model, using the kinetic theory of gases. This model is also suitable for dilute traffic conditions only 
and assumes that the desired speeds of the drivers are independent of the local traffic conditions. Phillips [29] employed statistical techniques used in the kinetic theory of gases to develop a model that produces results which remain stable over a wide range of traffic densities. Khan [20] used the ideal gas law and developed analogies of the gas parameters with traffic parameters. Accordingly, a driver's anticipation term was developed, which replaces the constant anticipation term in the PW model. Khan numerically solved the PW model and the proposed model using the Roe scheme [30]. Both models were then implemented and simulated in MATLAB for performance evaluation. Similarly, Imran et al. [31] also developed a traffic-flow model by deriving analogies between traffic flow and fluid flow, using Darcy's Law. This model attempts to characterize the heterogeneous traffic by replacing the constant anticipation term in the PW model. However, the values taken for traffic parameters in the proposed model also result in a constant term. The proposed model was simulated in MATLAB on a circular road, along with other models, and the results were compared.

In this study, a macroscopic model is developed for heterogeneous traffic conditions based on the gap-filling behavior. The model is derived by developing analogies of the traffic parameters with gas parameters used in the Maxwell-Boltzmann equation for the effusion of gases [32]. The performance of the proposed and PW models is then compared by using numerical simulations on a straight road (with and without ingress traffic) and a circular road.

The remainder of this paper is organized as follows. The development of the proposed model is presented in Section 3. The numerical solution of both the proposed and PW models is presented in Section 4. Finally, the numerical simulation of both models on straight (with and without ingress traffic) and circular roads is presented in Section 5, along with detailed discussion on each scenario. Section 6 draws pertinent conclusions and presents future work.

\section{Proposed Traffic-Flow Model}

Diffusion of gases is defined as the movement of gas particles from a zone of higher concentration to a zone of lower concentration. Effusion is the diffusion of gas particles but only through an orifice. The number of particles, $N_{c}$, effused out from a container through an orifice in a unit time is given by the following [32]:

$$
\frac{d N_{c}}{d t}=\frac{P A N_{A}}{\sqrt{2 \pi M R T}}
$$

where $P$ is the pressure of gas, $A$ is the cross-sectional area of the orifice through which the gas particles effuse out, $N_{A}$ is Avogadro's Number, $M$ is the molar mass of gas, $R$ is the gas constant, and $T$ is the temperature.

We assume that the vehicles have similar behavior to that of the gas particles inside a container. According to the kinetic theory of gases, the particles move freely until they collide with other particles. Similarly, a vehicle moves freely until it interacts with another vehicle. Moreover, the lateral spacing between the front vehicles is analogous to the crosssectional area of the orifice in the container. By this approach, analogies are developed, and a traffic-flow model is derived as outlined below and listed in Table 1.

Table 1. Analogies of gas parameters with traffic parameters.

\begin{tabular}{cc}
\hline Gas Parameters & Traffic-Flow Parameters \\
\hline $\mathrm{P}$ & $\rho \theta$ \\
$\mathrm{A}$ & $\mathrm{B}$ \\
$\mathrm{M}$ & $\rho$ \\
$\mathrm{RT}$ & $\rho \bar{v}^{2}$ \\
$N_{A}$ & $\rho$ \\
\hline
\end{tabular}


Traffic pressure is analogous to gas pressure and is equal to the product of traffic density and variance in velocity [29]:

$$
P=\rho \theta,
$$

where $\rho$ is the traffic density and $\theta$ is the variance in velocity. The greater the density, the greater will be the interaction between the vehicles. Similarly, the higher the variance in velocity, the higher will be the interaction, and, thus, the higher will be the traffic pressure.

As mentioned earlier, the cross-sectional area of the orifice is assumed to be analogous to the lateral headway between the front vehicles. Thus, we obtain the following:

$$
A=b,
$$

where $b$ is the lateral headway between the vehicles in the front.

According to Graham's law of effusion, the rate of movement of molecules is inversely proportional to the square root of the molar mass [32]. Molar mass can be taken as analogous to the traffic density. For a greater number of vehicles (higher traffic density), the flow is congested, and movement is limited. Conversely, for lesser number of vehicles, the flow is uniform, and the movement is relatively free. Thus, we obtain the following:

$$
M=\rho .
$$

Now, for the gas constant and absolute temperature, according to the fluid internal energy equation, the kinetic energy is given as follows:

$$
K . E=\frac{1}{2} M \bar{v}^{2},
$$

Since vehicular flow is in only one dimension, we can replace $\bar{v}$ by $\bar{v}_{x}$ and express Equation (7) as follows:

$$
K . E=\frac{1}{2} M \bar{v}_{x}^{2},
$$

For gases, it is established that the average velocity in one dimension is given as follows:

$$
\bar{v}_{x}^{2}=\frac{R T}{M},
$$

Thus, this can be further expressed as follows:

$$
\frac{1}{2} M \bar{v}^{2}=\frac{1}{2} R T,
$$

According to Equation (6), for traffic flow, the molar mass can be replaced with the traffic density:

$$
R T=\rho \bar{v}^{2},
$$

This means that the product of the gas constant and temperature in Equation (3) can be replaced with the product of traffic density and square of the average velocity.

Avogadro's Number is defined as the number of particles of a substance per unit mole of that substance. It is used to model the behavior of gas on a macroscopic level [33].

$$
N_{A}=\frac{\text { No. of Particles Accomodated }}{\text { Mole }}
$$

This is analogous to the number of vehicles that can be accommodated in a road section having a particular capacity.

$$
N_{A}=\frac{\text { No. of Vehicles Accomodated }}{\text { Capacity }}
$$


The road capacity can be represented by the average headway since the average headway is constant for a particular road capacity, while the number of vehicles accommodated in that particular road section under the said road capacity can be represented by the traffic density. Hence, this can be expressed as follows:

$$
N_{A}=\frac{\rho}{l}
$$

where $l$ is the average distance headway.

After developing analogies for gas parameters in Equation (3) with traffic parameters (see Table 1), substituting Equations (4)-(6), (11), and (14) in Equation (3), we get the following:

$$
\frac{d N_{C}}{d t}=\frac{b \cdot \theta}{\bar{v} \sqrt{2 \pi}} \cdot \frac{\rho}{l}
$$

The left-hand side of Equation (15) has the units of number of cars (or vehicles) per unit time, i.e., the traffic flow. Dividing $l$ on both sides, and knowing that $N_{C} / l=\rho$ and $b / v=t$ (time headway), Equation (15) can be rewritten as follows:

$$
\frac{d \rho}{d t}=\frac{\mathrm{t} \theta}{\sqrt{2 \pi}} \frac{\rho}{l^{2}}
$$

Multiplying $\rho$ on both sides, re-arranging, and taking the integral on both sides, we get the following:

$$
\frac{\rho^{2}}{2}=\frac{t^{2} \theta}{2 \sqrt{2 \pi}} \frac{\rho^{2}}{l^{2}}
$$

Rearranging for $\theta$, we can further establish the following:

$$
\theta=\frac{l^{2} \sqrt{2 \pi}}{t^{2}}
$$

This variance in velocity is due to the drivers' anticipation of filling the gaps seen ahead of them. It has units of $(\mathrm{m} / \mathrm{s})^{2}$ and depends on the average distance and time headway of the traffic. The proposed model is a macroscopic traffic-flow model based on the gap-filling behavior of heterogeneous traffic. It is derived by replacing the driver's anticipation constant $C_{0}$ in the PW model (Equation (2)). The proposed model can be written as follows:

$$
\begin{gathered}
\frac{\partial \rho}{\partial t}+\frac{\partial(\rho v(\rho))}{\partial x}=0 \\
\frac{\partial v}{\partial t}+\frac{v \partial v}{\partial x}=-\frac{l^{2} \sqrt{2 \pi}}{\rho t^{2}} \frac{\partial \rho}{\partial x}+\frac{v(\rho)-v}{\tau}
\end{gathered}
$$

Therefore, instead of assuming a constant behavior for all the drivers in the traffic (as in PW model), the drivers, will now adjust to the forward changing density conditions based on the distance headway and time headway according to the proposed model. This aspect is a substantial improvement upon existing models.

\section{Numerical Analysis}

Partial Differential Equations (PDEs) having a hyperbolic nature may result in shockwaves. Therefore, these systems of equations must be approximated by using Finite Volume Methods (FVMs) [34]. One of these methods is the Roe's Scheme. In Roe's Scheme, the models are first written in conservation form. Since this research numerically simulates both the PW and the proposed models, these models can be written in conservation form, as follows:

PW model:

$$
(\rho)_{t}+(\rho v(\rho))_{x}
$$




$$
(\rho v)_{t}+\left(\rho v^{2}+C_{0}^{2} \rho\right)_{x}=\rho \frac{v(\rho)-v}{\tau}
$$

Proposed model:

$$
\begin{gathered}
(\rho)_{t}+(\rho v(\rho))_{x} \\
(\rho v)_{t}+\left(\rho v^{2}+\frac{l^{2} \sqrt{2 \pi}}{t^{2}} \rho\right)_{x}=\rho \frac{v(\rho)-v}{\tau}
\end{gathered}
$$

The generalized conservation form is given as:

$$
u_{t}+f(u)_{x}=S
$$

where $u$ is a vector of traffic variables, $f(u)$ is a vector of function of traffic variables, and $S$ is a source term. Initially, the source term is assumed to be zero, i.e., there is no relaxation. Therefore, Equation (25) can also be written as follows:

$$
\frac{\partial u}{\partial t}+A(u) \frac{\partial u}{\partial x}=0
$$

\begin{tabular}{|c|c|c|}
\hline Vectors & PW Model & Proposed Model \\
\hline$u$ & $\left.\begin{array}{c}\rho \\
\rho v\end{array}\right]$ & $\begin{array}{c}\rho \\
\rho v\end{array}$ \\
\hline$f(u)$ & $\begin{array}{c}\rho v \\
\rho v^{2}+C_{0}^{2} \rho\end{array}$ & $\begin{array}{c}\rho v \\
\rho v^{2}+\frac{l^{2} \sqrt{2 \pi}}{t^{2}} \rho\end{array}$ \\
\hline$S$ & {$\left[\begin{array}{c}0 \\
\rho \frac{v(\rho)-v}{\tau}\end{array}\right]$} & {$\left[\begin{array}{c}0 \\
\rho \frac{v(\rho)-v}{\tau}\end{array}\right]$} \\
\hline$A(u)$ & $\begin{array}{cc}0 & 1 \\
C_{0}^{2}-v^{2} & 2 v\end{array}$ & $\begin{array}{cc}0 & 1 \\
\frac{l^{2} \sqrt{2 \pi}}{t^{2}}-v^{2} & 2 v\end{array}$ \\
\hline$e_{1,2}$ & $\left.\begin{array}{c}1 \\
v \pm C_{0}\end{array}\right]$ & $v \pm \frac{1}{l^{2} \sqrt{2 \pi}}$ \\
\hline
\end{tabular}

where $A(u)$ is known as the Jacobian matrix. The vectors of the PW and proposed models are presented in Table 2.

Table 2. Vectors of the PW and proposed models.

where $e_{1,2}$ are the eigen vectors of the corresponding models. According to Roe's Scheme, the average velocity at the boundaries or edges of the cells is given as follows:

$$
v=\frac{\sqrt{\rho_{2}} v_{2}+\sqrt{\rho_{1}} v_{1}}{\sqrt{\rho_{2}}+\sqrt{\rho_{1}}}
$$

Furthermore, the average density at the boundaries or edges of cells is given as follows:

$$
\rho=\sqrt{\rho_{2} \rho_{1}}
$$

The generalized approach of Roe's Scheme is to divide the entire road segment into cells depending upon the road step, assuming that the total length of the road is $x$. Then the number of cells is given as $N=x / d x$, where $d x$ is the road step. The number of edges or boundaries of these road segments is equal to $N+1$. The total time of simulation is $T$, and the number of time steps is $T / d t$, where $d t$ is the time step. At the start, initial conditions for traffic density, as well as velocity, are assigned to each cell. Subsequently, the Jacobian matrix is approximated at the boundaries or edges, using the Roe's Scheme. 
Thereafter, the vector of the function of traffic variables $f(u)$ at the edge or boundary between the cells $i$ and $i+1$ at time $t$ is approximated by using the following equation:

$$
f_{i+\frac{1}{2}}^{t}\left(u_{i}^{t}, u_{i+1}^{t}\right)=\frac{1}{2}\left(f\left(u_{i}^{t}\right)+f\left(u_{i+1}^{t}\right)\right)-\frac{1}{2} A\left(u_{i+\frac{1}{2}}\right)\left(u_{i+1}^{t}-u_{i}^{t}\right)
$$

This way, the vectors of function of traffic variables are estimated at all the boundaries or edges between the cells. After that, the traffic variables are updated for the next time step, using the following equation:

$$
u_{i}^{t+1}=u_{i}^{t}-\frac{\mathrm{d} t}{\mathrm{~d} x}\left(f_{i+\frac{1}{2}}^{t}+f_{i-\frac{1}{2}}^{t}\right)+\mathrm{d} t \cdot S\left(u_{i}^{t}\right)
$$

where $S\left(u_{i}^{t}\right)$ is the source term at time $t$ and cell $i$. Accordingly, using initial boundary conditions, the model is numerically approximated at all the road segments and at every time step. In case of difference in values at the edges or boundaries of cells, there may be discontinuous results at the edges between the cells. For this reason, the entropy fix is applied [20]. According to the entropy fix, the Jacobian matrix is substituted with $e|\Lambda| e^{-1}$, where $|\Lambda|$ is a diagonal matrix, which is a function of the eigen values, while $e$ is the corresponding eigen vector. The entropy fix scheme employed by Harten and Hyman [35] is described as follows:

$$
\begin{gathered}
\lambda= \begin{cases}\delta, & |\lambda|<\delta \\
|\lambda|, & |\lambda| \geq \delta\end{cases} \\
\delta=\max \left(0, \quad \lambda_{i+\frac{1}{2}}-\lambda_{i} \quad \lambda_{i+1}-\lambda_{i+\frac{1}{2}}\right)
\end{gathered}
$$

\section{Simulation Results and Discussion}

The proposed model and the PW model are numerically simulated in MATLAB to evaluate and compare their performance. Both models are simulated for (i) straight road with ingress traffic, (ii) straight road with no ingress traffic, and (iii) circular road. The total length of the road for both models is selected as $100 \mathrm{~m}$, and the simulation is carried out for a total time of $30 \mathrm{~s}$ for a straight road with ingress traffic and circular road, and $2 \mathrm{~s}$ for a straight road with no ingress traffic. It can be noted that, since the traffic conditions become smooth and traffic becomes uniform (or the effect of the inactive bottleneck is diminished after sometime), there is no need for additional simulation time. The maximum velocity for both models is chosen as $25 \mathrm{~m} / \mathrm{s}(90 \mathrm{~km} / \mathrm{h})$, and the relaxation time is selected as $0.5 \mathrm{~s}$, which means that the equilibrium velocity is achieved in a short time. The maximum density is chosen as 1.0 because in the simulations the density used is the normalized density, which is equal to the ratio of the actual density to the maximum density. The time step and distance step are selected so that they satisfy the Courant-Friedrichs-Levy (CFL) [36] stability condition, according to which $(d t / d x) * v M a x \leq 1$. Following this condition, the maximum time step that could be chosen should be less than or equal to $0.04 \mathrm{~s}$.

The initial density conditions at $t=0$ are such that the density is 0.1 till the distance of $50 \mathrm{~m}$, while that from $50 \mathrm{~m}$ onwards is 0.6 (see Equation (33)), which means that there is a sudden increase in density.

$$
\rho= \begin{cases}0.1, & x \leq 50 \\ 0.6, & x>50\end{cases}
$$

This sudden increase in density can be physically thought of as a bottleneck. The initial velocity conditions are according to the Greenshields velocity distribution [37]. The input parameters for the simulation of both models are summarized in Table 3. 
Table 3. Input parameters for the numerical simulation of the proposed and PW models.

\begin{tabular}{cc}
\hline Input Parameters & Values \\
\hline Equilibrium velocity distribution & Greenshields \\
\hline Road length & $100 \mathrm{~m}$ \\
Distance step & $1 \mathrm{~m}$ \\
\hline Simulation time for: & $2 \mathrm{~s}$ \\
Straight road, no ingress traffic & $30 \mathrm{~s}$ \\
Straight road, ingress traffic & $30 \mathrm{~s}$ \\
Circular road & $0.01 \mathrm{~s}$ \\
Time step & $0.5 \mathrm{~s}$ \\
Relaxation time & $0.5 \mathrm{~s}$ \\
Time headway for proposed model & $25 \mathrm{~m} / \mathrm{s}(90 \mathrm{~km} / \mathrm{h})$ \\
PW model anticipation constant & $25 \mathrm{~m} / \mathrm{s}(90 \mathrm{~km} / \mathrm{h})$ \\
\hline Maximum velocity & 1 \\
Maximum density &
\end{tabular}

\subsection{Straight Road with Ingress Traffic}

For straight road with ingress traffic, the ingress traffic follows a cosine function with densities ranging between 0.25 to 0.5 and velocities following the Greenshields distribution. The 3D plot for the PW model is depicted in Figure 1. The PW model does not exhibit any unrealistic behavior for densities, that is the densities at all the time instants and at all road steps are well within the defined range. However, it can be observed that, despite the very high initial densities and ingress traffic, the density curve smoothens very quickly, and no significant effect of the bottleneck is translated at any road segment over the entire simulation period.

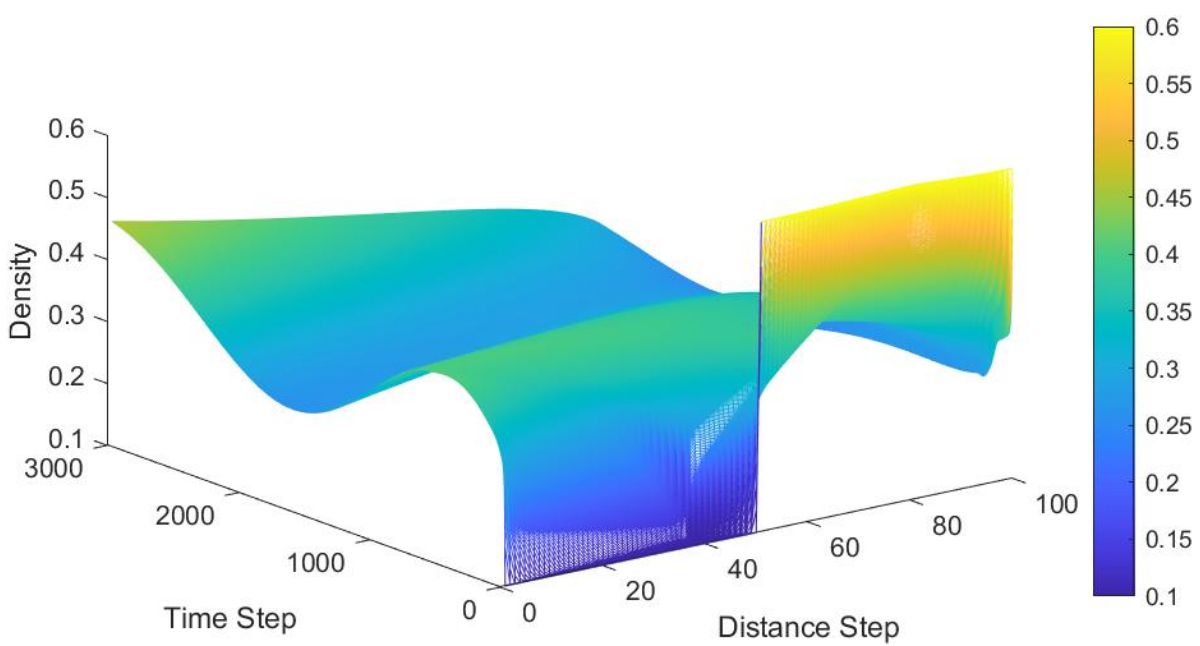

Figure 1. Density behavior of PW model on straight road with ingress traffic.

The velocity behavior of the PW model is illustrated in Figures 2 and 3. As discussed earlier, because of the constant anticipation term, the PW model results in negative velocities, which is not possible. Another unrealistic behavior is that the PW model exhibits velocities higher than the maximum velocity. Out of bound velocities have been highlighted in red in Figure 2. It can be further observed in Figure 3 that the problem of negative velocities persists till $0.35 \mathrm{~s}$, while velocities exceeding the maximum velocity can be observed till $1.66 \mathrm{~s}$, where the velocity is just above $25 \mathrm{~m} / \mathrm{s}(90 \mathrm{~km} / \mathrm{h})$. At very early time instants, the velocities reach as high as $35.4 \mathrm{~m} / \mathrm{s}(127 \mathrm{~km} / \mathrm{h})$. Beyond that, the velocities are well within the defined range. 


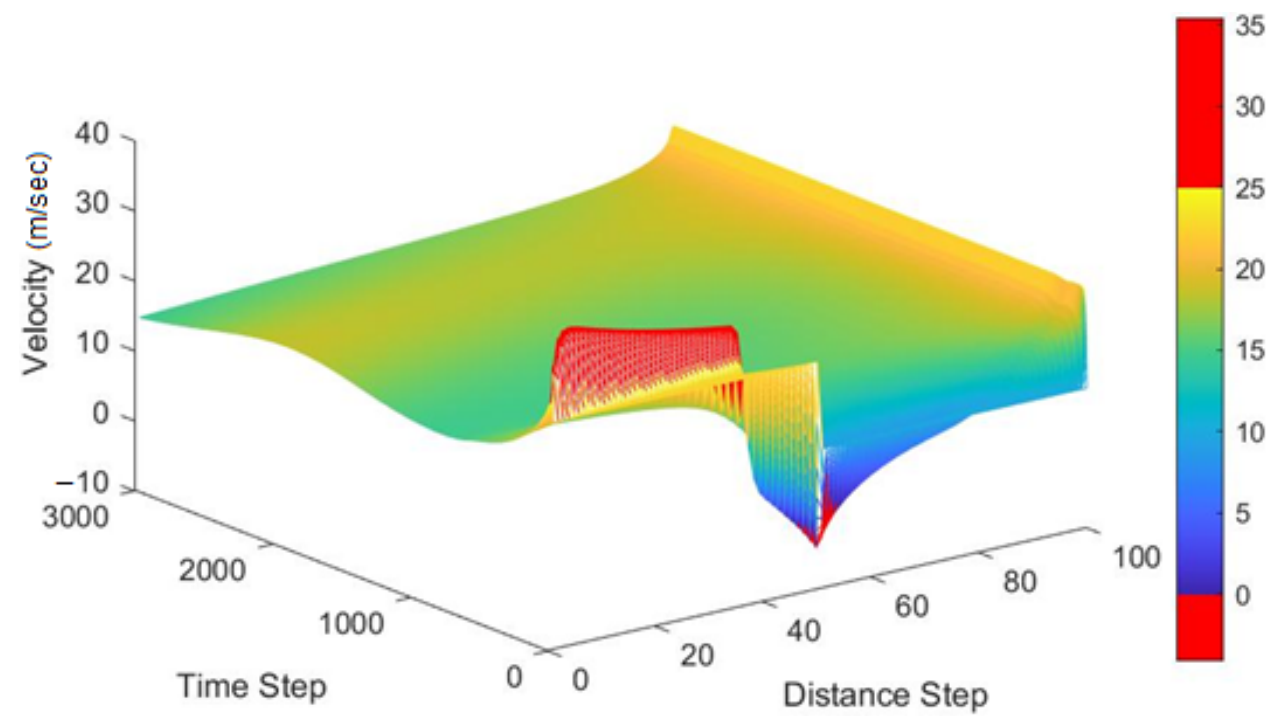

Figure 2. Velocity behavior of PW model on straight road with ingress traffic.

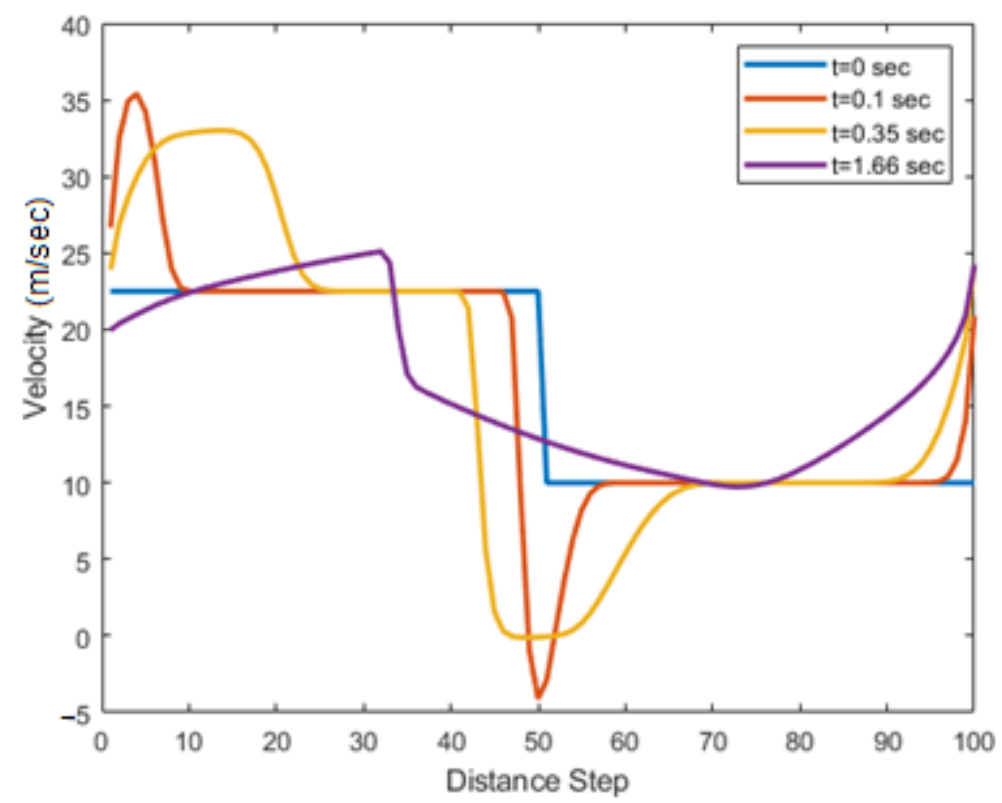

Figure 3. Velocity behavior of PW model on straight road with ingress traffic.

When simulated for similar traffic conditions, the proposed model exhibits robust and realistic results. In the case of density, it can be observed that the effect of the bottleneck has been projected backwards and an increasing density can be observed beyond the bottleneck (see Figures 4 and 5). At time $t=15 \mathrm{~s}$, the density even reaches a value of 0.67 . After that, the effect of the bottleneck starts diminishing steadily, as can be seen at time $t=17 \mathrm{~s}$.

The velocity behavior of the proposed model is even better. There is no unrealistic behavior at any road segment throughout the simulation period and the velocities are well within the defined range. As can be observed in Figures 6 and 7, the velocity starts increasing as the density decreases, which indicates that more gaps are produced ahead, and, hence, the drivers are encouraged to fill those gaps, resulting in the said increase of velocity. 


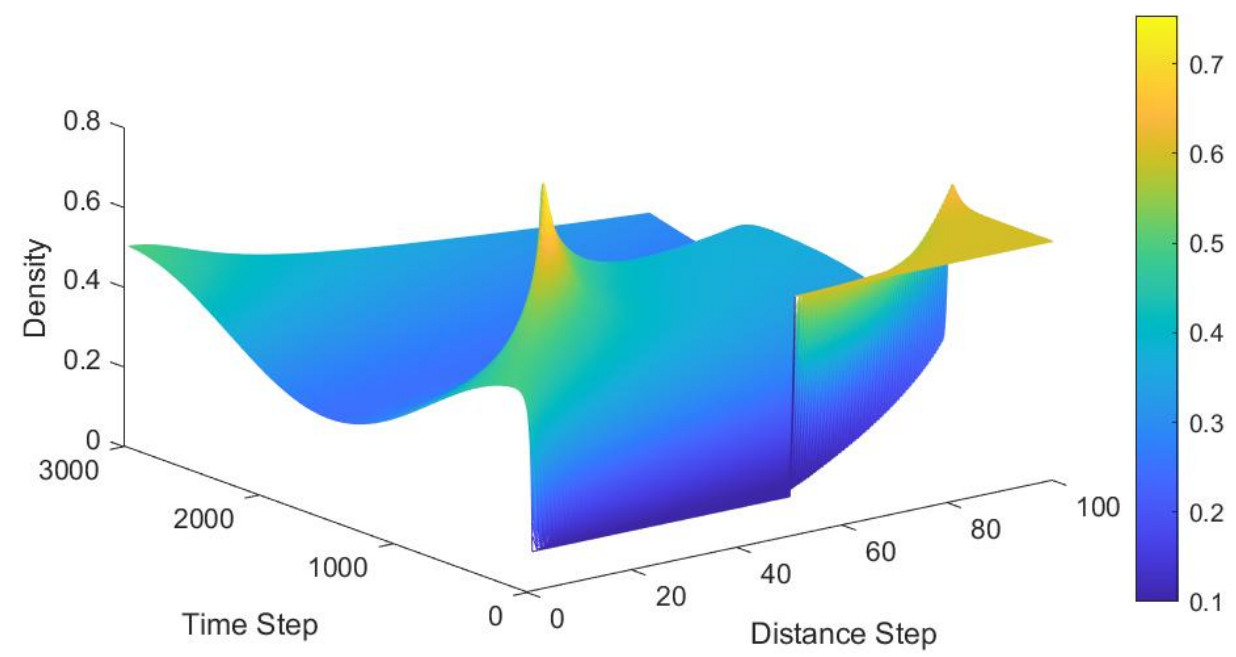

Figure 4. Density behavior of proposed model on straight road with ingress traffic.

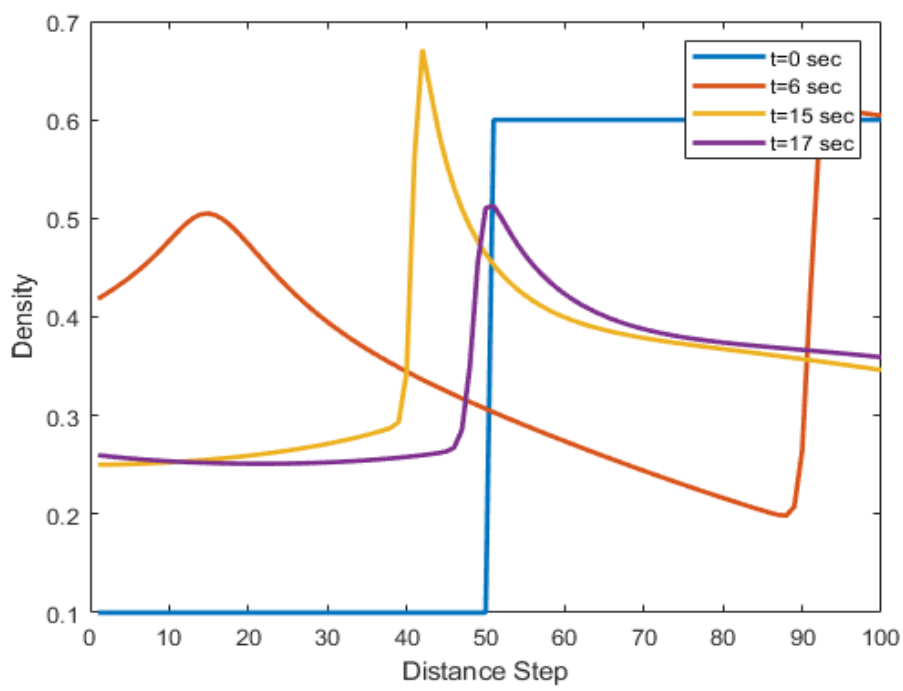

Figure 5. Density behavior of proposed model on straight road with ingress traffic.

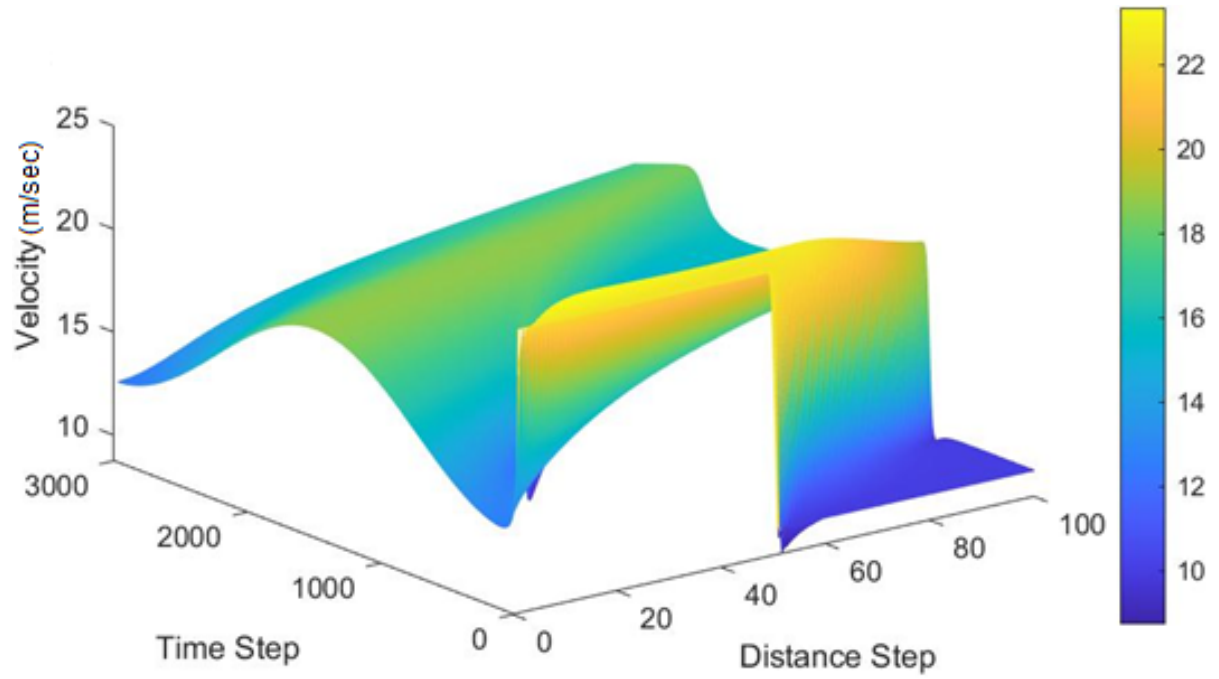

Figure 6. Velocity behavior of proposed model on straight road with ingress traffic. 


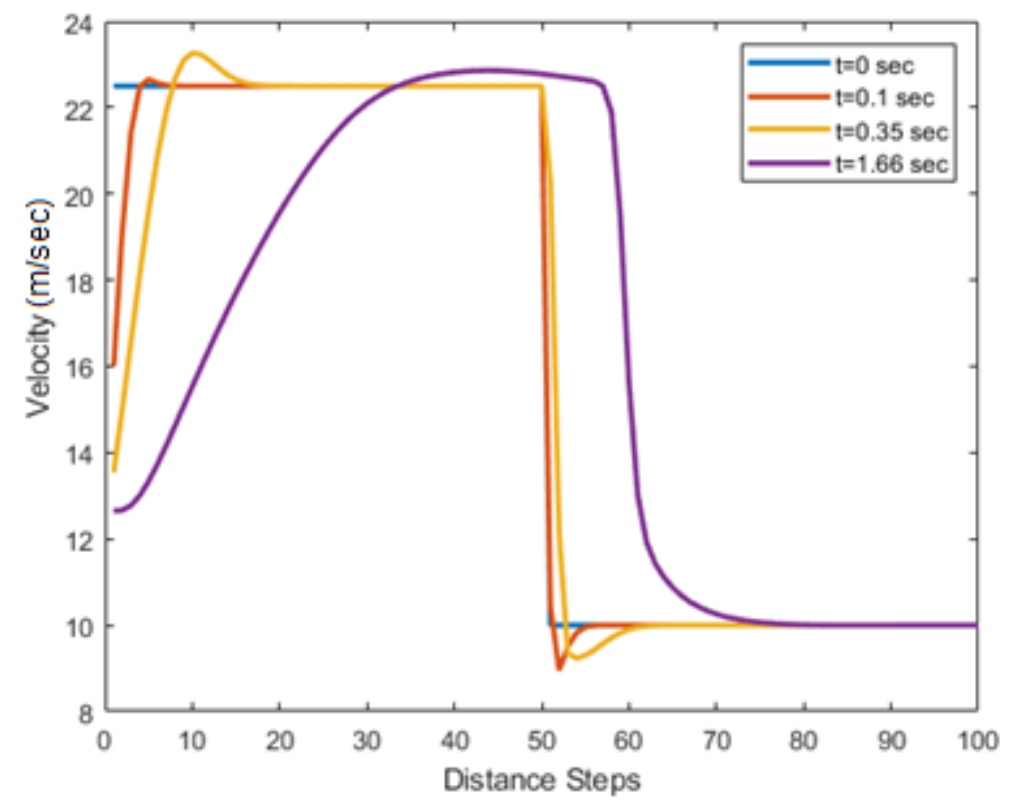

Figure 7. Velocity behavior of proposed model on straight road with ingress traffic.

\subsection{Straight Road with No Ingress Traffic}

In this case, there is no incoming traffic, and the existing traffic, which has been introduced to the road section as an initial condition, is leaving the road segment at a very high velocity. The total simulation time, in this case, was reduced to just $2 \mathrm{~s}$. This is because, after a few seconds, all the traffic has left the road section and no vehicles are left on the road. A sudden vacuum is produced, resulting in negative densities, which is not possible. First, the PW model is simulated. The density behavior is shown in Figure 8, which is well within the defined range. However, a very interesting observation can be made here. Despite all the traffic leaving the road section and no vehicles entering it, the density is observed to increase at some distance steps with the passage of time, which is unrealistic. The velocity behavior of the PW model is shown in Figures 9 and 10. Again, the negative velocities, which are highlighted in red in Figure 9, can be observed. Velocities get as low as $-4.1 \mathrm{~m} / \mathrm{s}(14.76 \mathrm{~km} / \mathrm{h})$ at $0.1 \mathrm{~s}$. The negative velocities are exhibited till $0.35 \mathrm{~s}$. In this case, however, the maximum velocity remains within the defined range and the model does not display any unrealistic behavior as far as the maximum velocity is concerned.

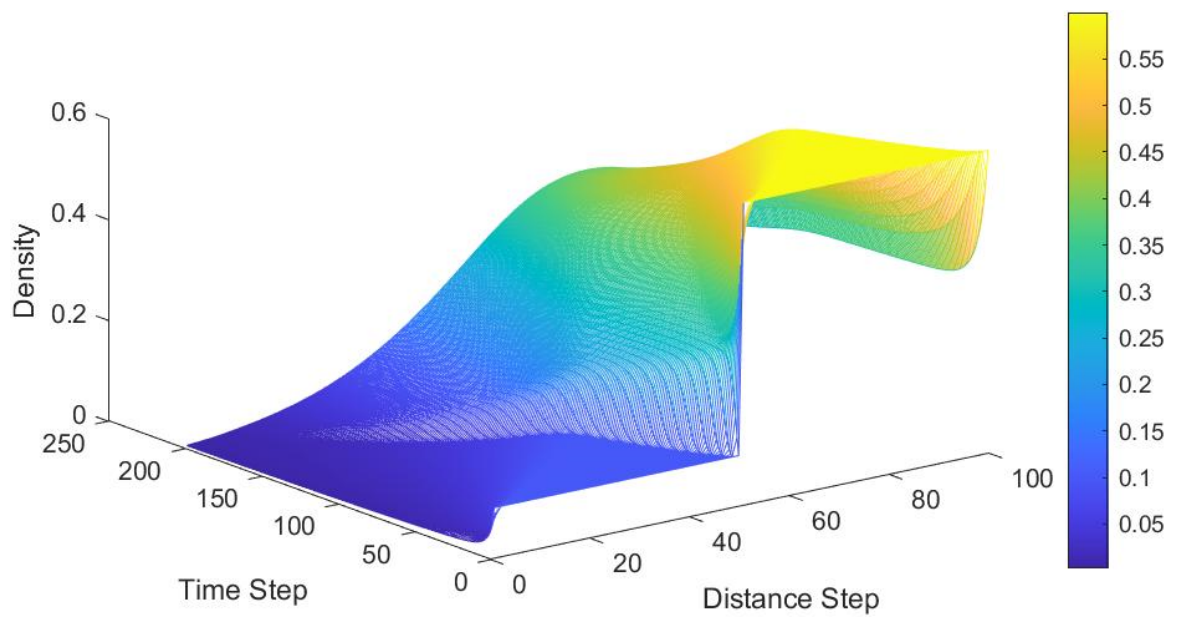

Figure 8. Density behavior of PW model on straight road with no ingress traffic. 


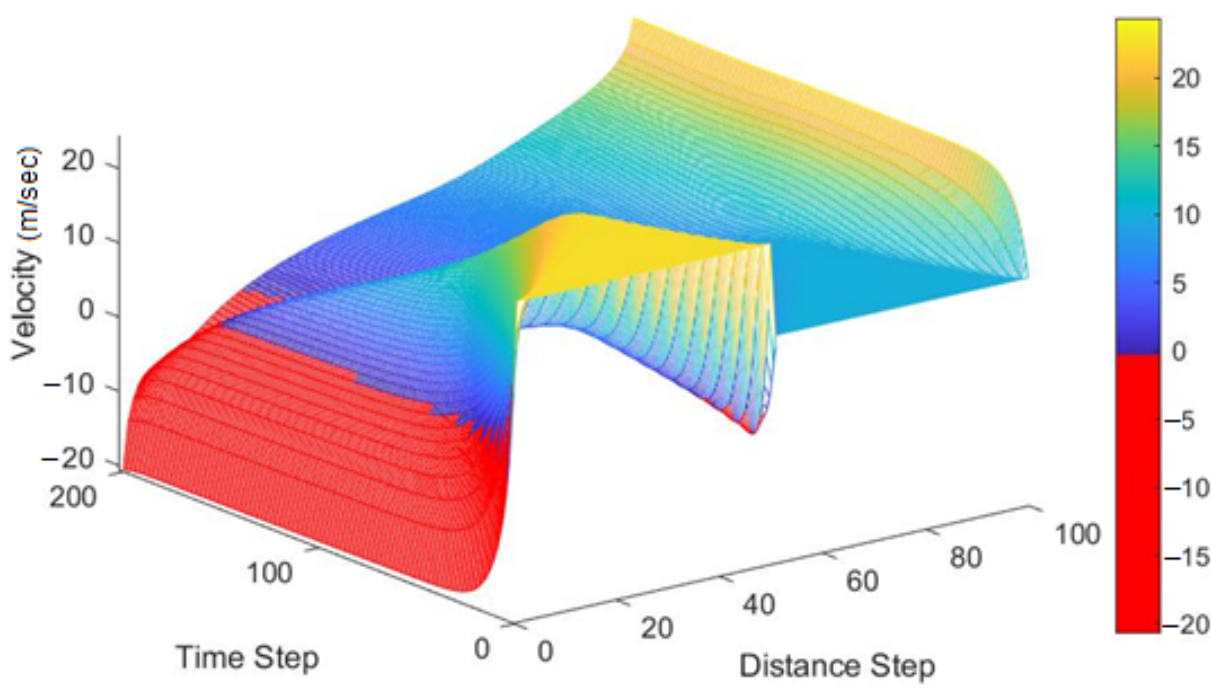

Figure 9. Velocity behavior of PW model on straight road with no ingress traffic.

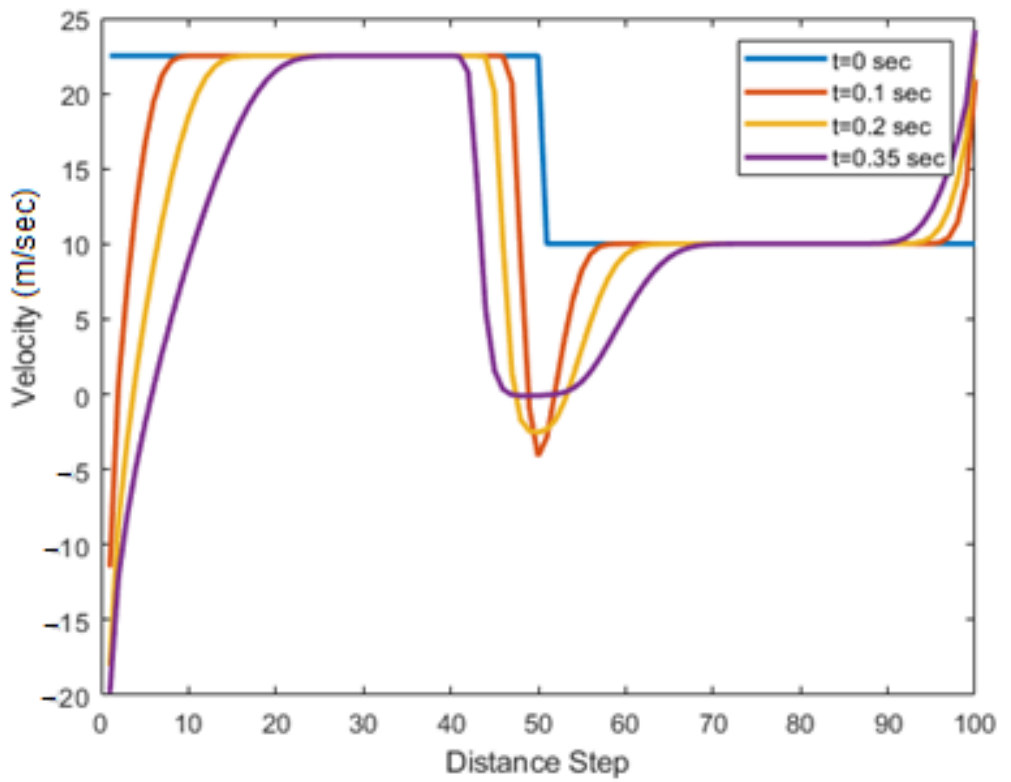

Figure 10. Velocity behavior of PW model on straight road with no ingress traffic.

Conversely, when simulated for similar conditions, the proposed model produces robust and realistic density and velocity results, which are within the defined ranges. The density behavior can be observed in Figure 11, while the velocity behavior is depicted in Figures 12 and 13. In the case of velocity, unlike the PW model, there is no negative velocity at any instant of time despite the considerable traffic density. The negative velocities, highlighted in red in Figure 12 and observed towards the end of the simulation period at the start of the road section, are rather meaningless, since there is no traffic density, implying that there are no vehicles on the road at that moment on that particular road section.

\subsection{Circular/Ring Road}

For the case of a circular road, the simulation conditions were set such that the traffic parameters at the end of the road section were re-implemented at the entrance of the road section at the next time step, resulting in an oscillatory behavior, which is the physical equivalent of a circular or ring road. The density behavior of the PW model can be observed in Figure 14. It is almost identical to the behavior of the PW model on a straight road with traffic ingress. The density smoothens very quickly, and no significant effect of the 
bottleneck introduced in the initial conditions can be observed throughout the road section at all the other time intervals. Other than that, the density behavior is realistic and is well within the defined range.

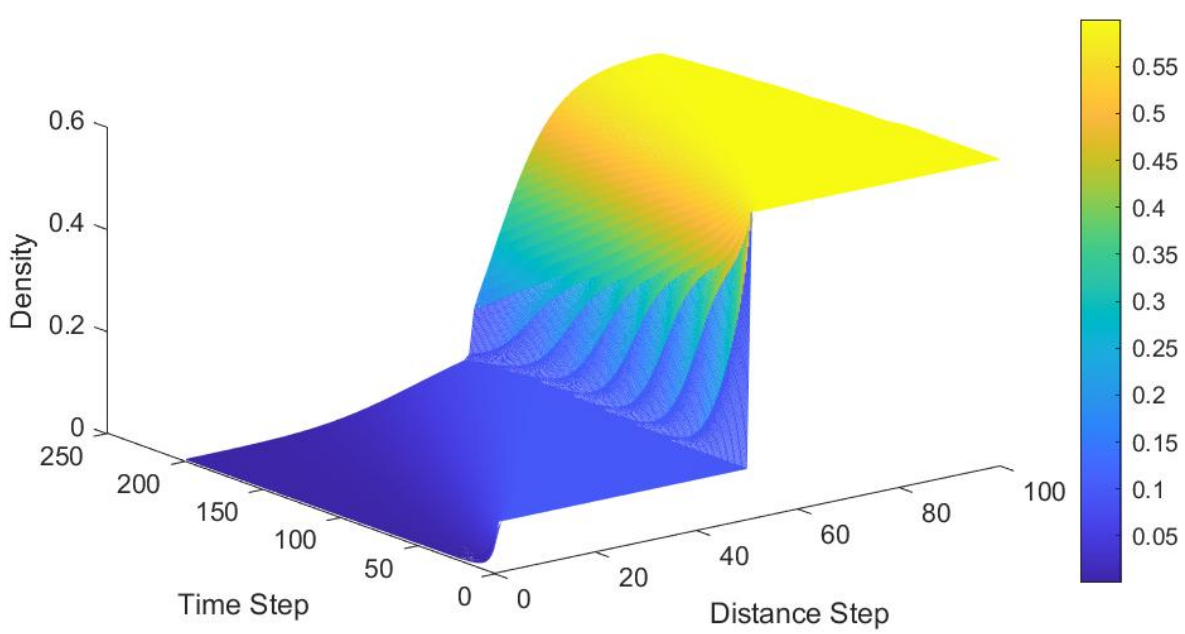

Figure 11. Density behavior of proposed model on straight road with no ingress traffic.

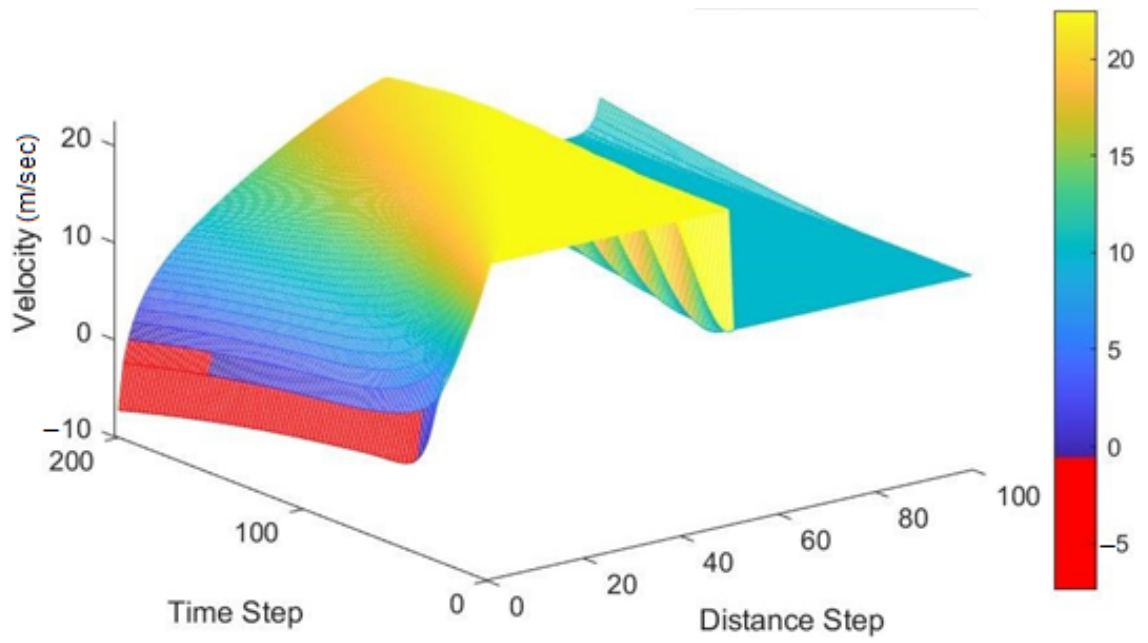

Figure 12. Velocity behavior of proposed model on straight road with no ingress traffic.

For the case of the velocity, it is almost identical to the velocity behavior observed for the straight road with ingress traffic as can be observed in Figures 15 and 16. The negative velocities are highlighted in red in Figure 15 . Velocities go as low as $-4.1 \mathrm{~m} / \mathrm{s}(14.76 \mathrm{~km} / \mathrm{h})$ and as high as $36.3 \mathrm{~m} / \mathrm{s}(130.68 \mathrm{~km} / \mathrm{h})$, both of which are unrealistic, as they are beyond the defined range. Negative velocities are observed until $0.35 \mathrm{~s}$, while the upper bound unrealistic velocities are observed until $1.66 \mathrm{~s}$. Beyond that, the model exhibits velocities within the defined range.

On the other hand, when simulated on the same ring road, the proposed model exhibited better and realistic results than that of the PW model. For the case of density, the effect of the bottleneck does not diminish suddenly. Rather, it leaves an impact, which can be observed in the form of a wave travelling in the backward direction, as can be observed in Figure 17. The effect of the bottleneck can be further observed in Figure 18, at 6, 10, 15, and $20 \mathrm{~s}$. An increased density can be observed at different time instants and at different distances, which can be seen travelling forward and reducing with time. This behavior can be considered closer to the anticipated reality for the case of heterogeneous traffic than that exhibited by the PW model. 


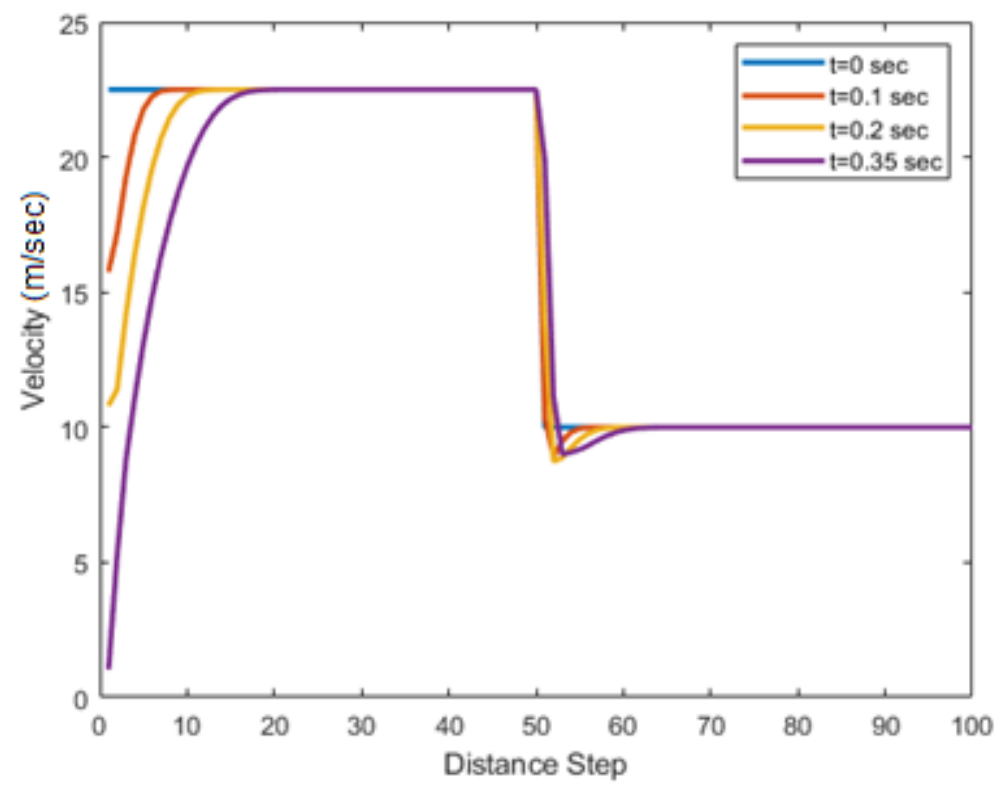

Figure 13. Velocity behavior of proposed model on straight road with no ingress traffic.

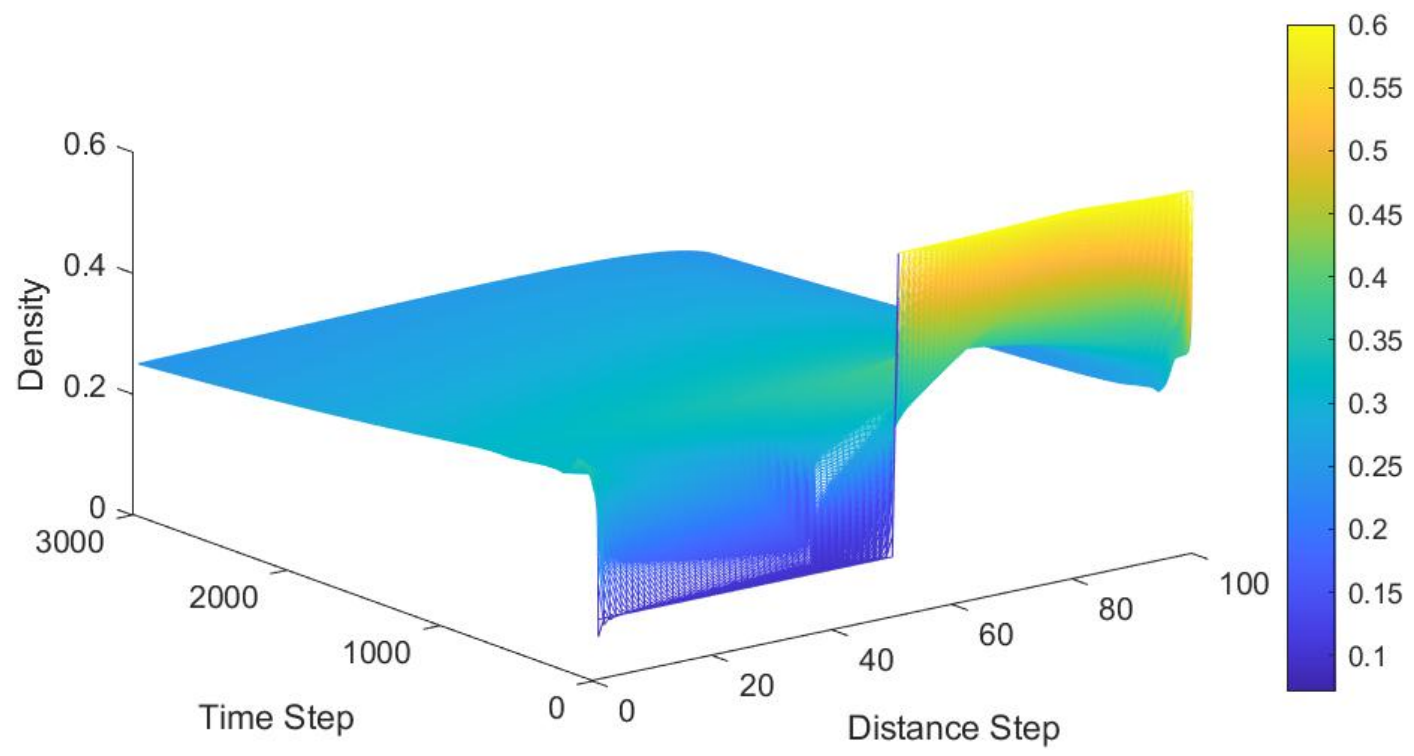

Figure 14. Density behavior of the PW model on a ring road.

As far as the velocity behavior is concerned, it is somewhat similar to the one observed in the case of the straight road with traffic ingress. Velocities at all points and all-time instants are within the defined range. As soon as there is a decrease in density, the velocity increases, which, again, enforces the gap-filling behavior: with the decrease in density, more gaps appear ahead of the vehicles, which causes the vehicles to rush towards them in order to occupy those gaps. The velocity behavior of the proposed model can be observed in Figures 19 and 20. 


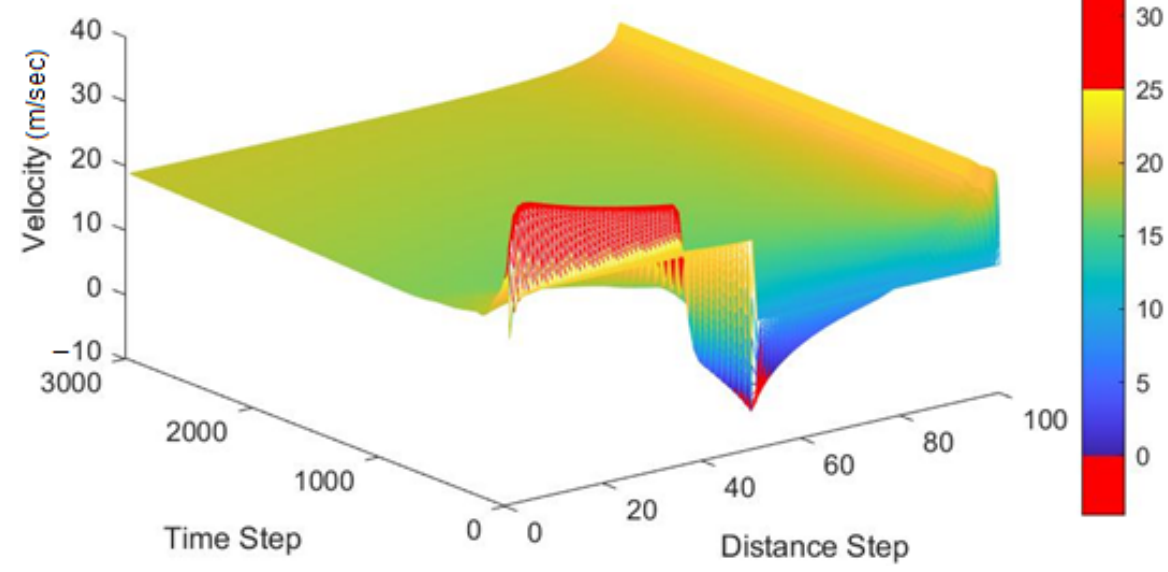

Figure 15. Velocity behavior of the PW model on a ring road.

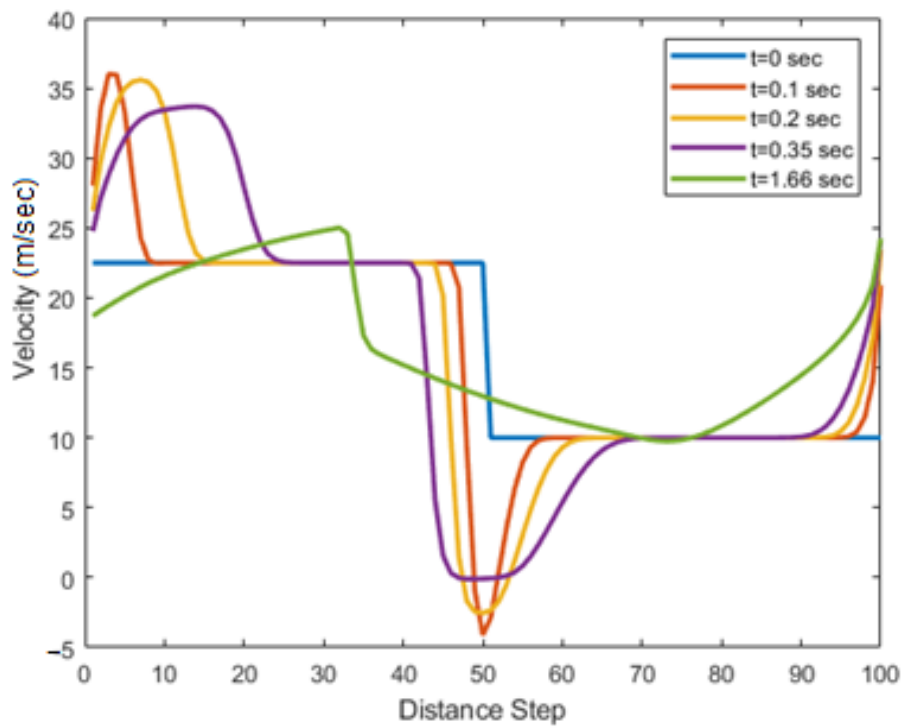

Figure 16. Velocity behavior of the PW model on a ring road.

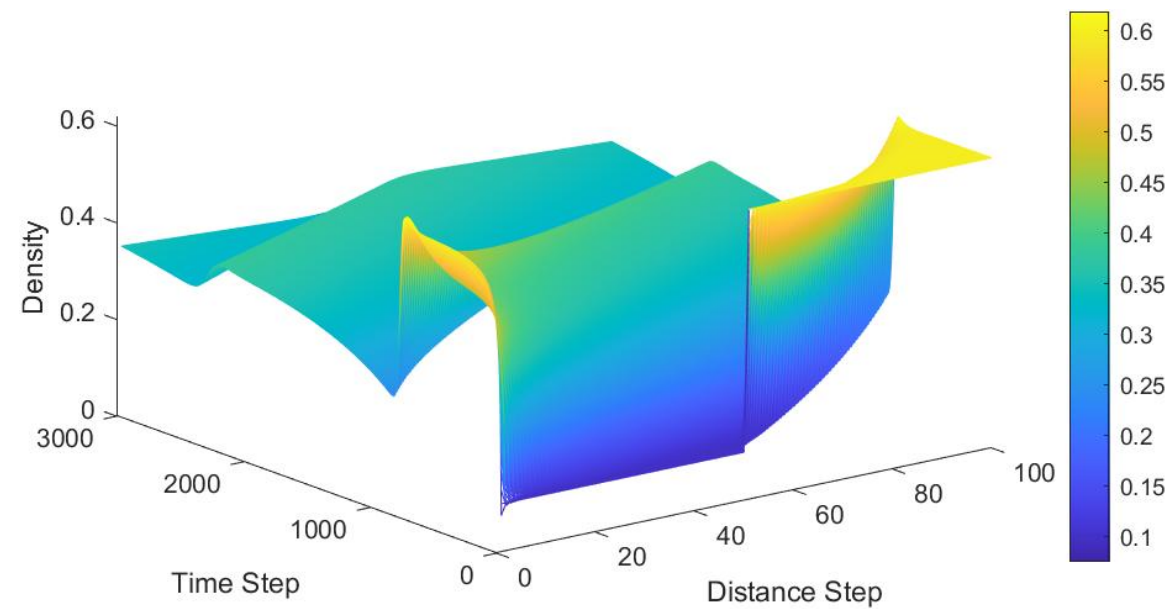

Figure 17. Density behavior of the proposed model on a ring road. 


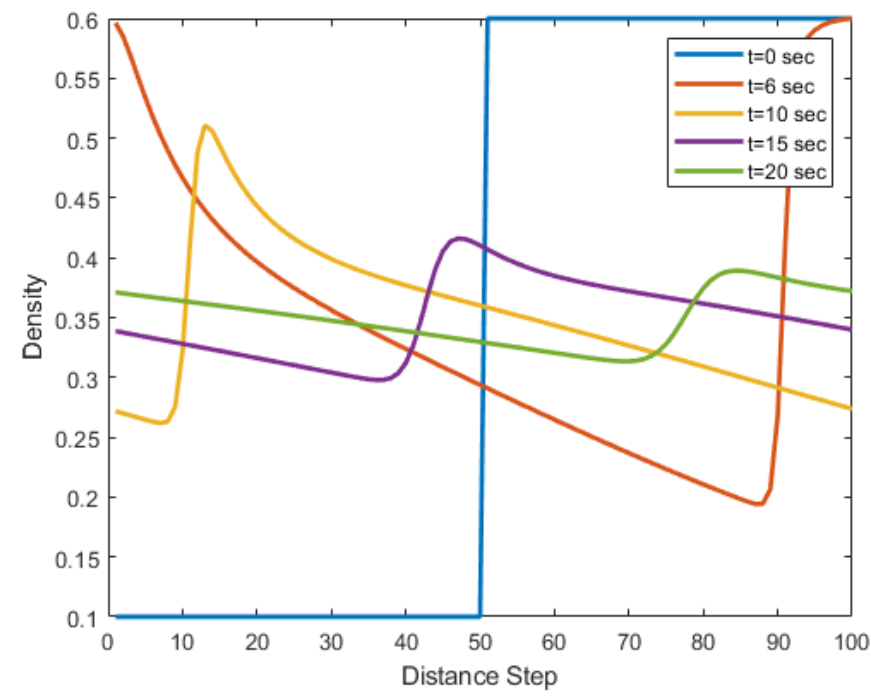

Figure 18. Density behavior of the proposed model on ring road.

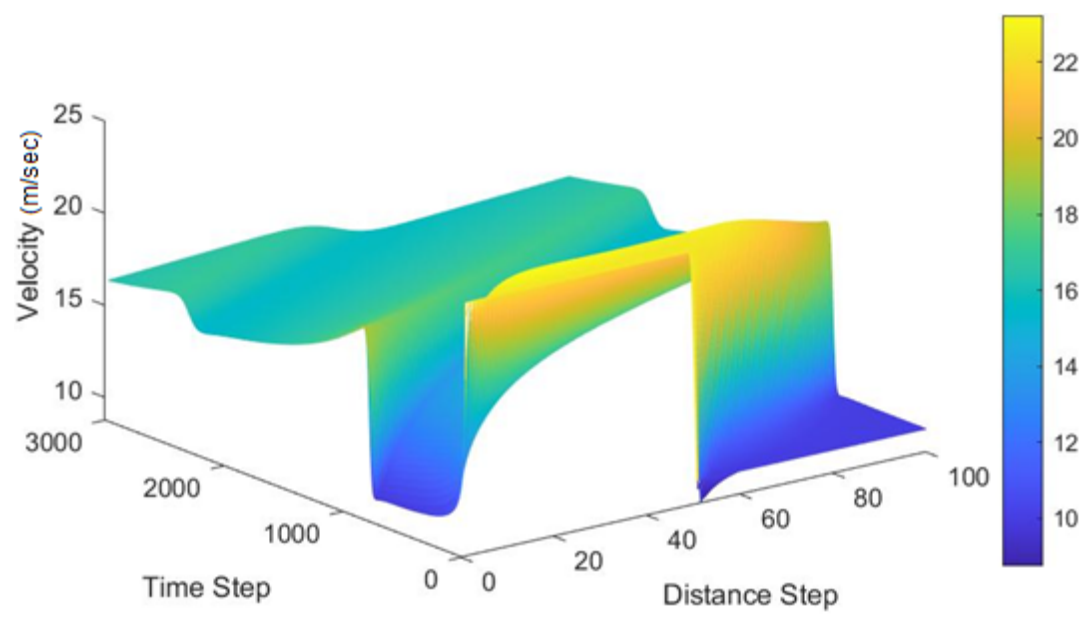

Figure 19. Velocity behavior of the proposed model on a ring road.

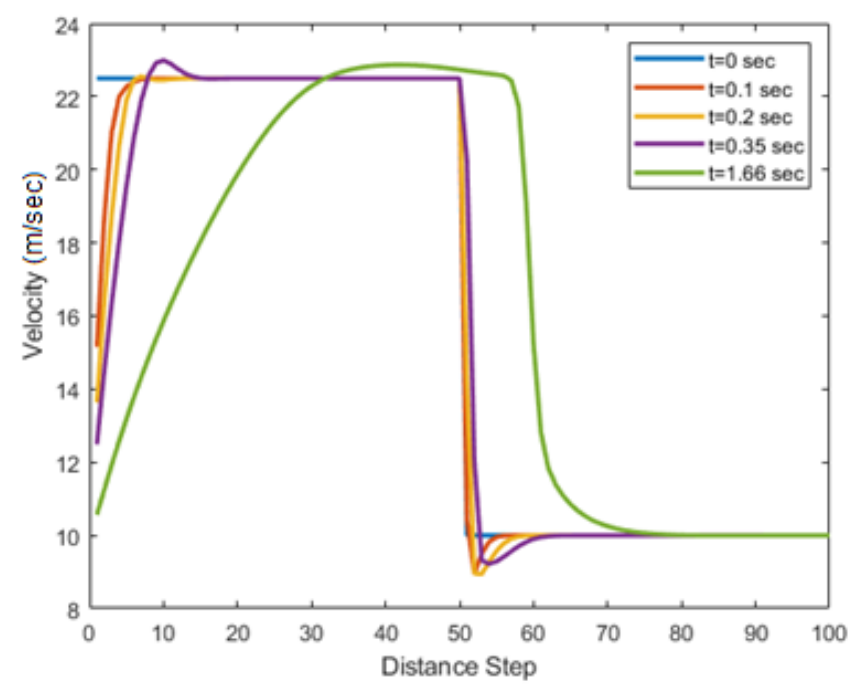

Figure 20. Velocity behavior of the proposed model on a ring road. 


\section{Conclusions and Future Work}

In heterogeneous traffic conditions, the drivers tend to fill the gaps between the vehicles in front, irrespective of the lane-discipline, causing extreme interactions between the vehicles. This unique behavior results in severe disturbances within the traffic stream, which is why the driver behavior leading to it must be characterized.

In this paper, a new macroscopic traffic-flow model based on the gap-filling behavior of heterogeneous traffic was proposed. In the first part of this study, a driver's anticipation term was derived that explains the gap-filling behavior in heterogeneous traffic. This anticipation term is derived by developing analogies of traffic parameters with gas parameters, using the Effusion model of the gases. Then, a model for heterogeneous traffic was proposed by replacing the constant driver's anticipation term in the PW model with the derived model for the gap-filling behavior. The second part of this study comprised the numerical approximation of the proposed model, using the Roe Scheme. Finally, in order to observe the behavior of the proposed model, it was numerically simulated in MATLAB for three different types of road conditions: straight road with ingress traffic, straight road with no ingress traffic, and ring road or circular road. The results of the proposed model were further compared with those of the PW model. The PW model results in negative velocities and violates the upper velocity bound, as well. Moreover, the PW model does not significantly reflect the effect of the bottleneck introduced in initial conditions. On the other hand, the proposed model addresses the shortcomings of the PW model, attains robust and realistic results, and does not exhibit unrealistic velocities. It can be concluded that, unlike the PW model, the proposed model produces results that better simulate the expected reality, owing to the effect of the bottleneck which is projected backwards at other time instants in the form of a propagated shockwave.

For future research, an extension of this study would include the calibration and validation of the proposed model with real traffic data before it could be practically implemented. Moreover, it is planned to develop a new methodology in order to introduce different traffic conditions in the simulations of the macroscopic traffic-flow models. These traffic conditions may include active bottlenecks, traffic signals, intersections, and mergers. The authors are also planning to use different velocity distributions within different macroscopic traffic-flow models and then further comparing the results.

Author Contributions: M.U.K., conceptualization, methodology, software, formal analysis, data curation, visualization, and writing-original draft preparation; S.S., methodology, software, formal analysis, visualization, and writing-review and editing; M.L.N., validation, resources, project administration, funding acquisition, and writing-review and editing; R.R., project administration and review and editing. All authors have read and agreed to the published version of the manuscript.

Funding: This research received no external funding.

Institutional Review Board Statement: Not applicable.

Informed Consent Statement: Not applicable.

Conflicts of Interest: The authors declare no conflict of interest.

\section{References}

1. Kindberg, T.; Chalmers, M.; Paulos, E. Urban Computing: Concepts, Methodologies, and Applications. IEEE Pervasive Comput. 2007, 6, 18-20. [CrossRef]

2. Timilsina, G.R.; Dulal, H.B. Urban road transportation externalities: Costs and choice of policy instruments. World Bank Res. Obs. 2011, 26, 162-191. [CrossRef]

3. Trubia, S.; Severino, A.; Curto, S.; Arena, F.; Pau, G. Smart roads: An overview of what future mobility will look like. Infrastructures 2020, 5, 107. [CrossRef]

4. Pappalardo, G.; Cafiso, S.; Di Graziano, A.; Severino, A. Decision tree method to analyze the performance of lane support systems. Sustainability 2021, 13, 846. [CrossRef]

5. Vallati, M.; Magazzeni, D.; De Schutter, B.; Chrpa, L.; McCluskey, T.L. Efficient macroscopic urban traffic models for reducing congestion: A PDDL+ planning approach. In Proceedings of the 30th AAAI Conference on Artificial Intelligence AAAI 2016, Phoenix, AZ, USA, 12-17 February 2016; pp. 3188-3194. 
6. Zambrano-Martinez, J.L.; Calafate, C.T.; Soler, D.; Cano, J.C.; Manzoni, P. Modeling and characterization of traffic flows in urban environments. Sensors (Switzerland) 2018, 18, 2020. [CrossRef] [PubMed]

7. Bando, M.; Hasebe, K.; Nakayama, A.; Shibata, A.; Sugiyama, Y. Dynamical model of traffic congestion and numerical simulation. Phys. Rev. E 1995, 51, 1035-1042. [CrossRef] [PubMed]

8. Chandler, R.E.; Herman, R.; Montroll, E.W. Traffic Dynamics: Studies in Car Following. Oper. Res. 1958, 6, 165-184. [CrossRef]

9. Lighthill, M.J.; Whitham, G.B. On kinematic waves II. A theory of traffic flow on long crowded roads. Proc. R. Soc. London. Ser. A Math. Phys. Sci. 1955, 229, 317-345. [CrossRef]

10. Richards, P.I. Shock Waves on the Highway. Oper. Res. 1956, 4, 42-51. [CrossRef]

11. Payne, H.J. Models of freeway traffic and control. Math. Model. Publ. Syst. 1971, 1, 51-61.

12. Whitham, G. Linear and Nonlinear Waves; Wiley: New York, NY, USA, 1971.

13. Jamshidnejad, A.; Papamichail, I.; Papageorgiou, M.; De Schutter, B. A mesoscopic integrated urban traffic flow-emission model. Transp. Res. Part C Emerg. Technol. 2017, 75, 45-83. [CrossRef]

14. Tiwari, G.; Fazio, J.; Gaurav, S. Traffic planning for non-homogeneous traffic. Sadhana Acad. Proc. Eng. Sci. 2007, 32, 309-328. [CrossRef]

15. Mathew, T.V.; Radhakrishnan, P. Calibration of microsimulation models for nonlane-based heterogeneous traffic at signalized intersections. J. Urban Plan. Dev. 2010, 136, 59-66. [CrossRef]

16. Arasan, V.T.; Koshy, R.Z. Methodology for modeling highly heterogeneous traffic flow. J. Transp. Eng. 2005, 131, 544-551. [CrossRef]

17. Khan, Z.H.; Gulliver, T.A. A Macroscopic Traffic Model Based on Anticipation. Arab. J. Sci. Eng. 2019, 44, 5151-5163. [CrossRef]

18. Daganzo, C.F. Requiem for second-order fluid approximations of traffic flow. Transp. Res. Part B 1995, 29, 277-286. [CrossRef]

19. Aw, A.; Rascle, M. Resurrection of "second order" models of traffic flow. SIAM J. Appl. Math. 2000, 60, 916-938. [CrossRef]

20. Khan, Z. Traffic Modelling for Intelligent Transportation Systems. Ph.D. Thesis, University of Victoria, Victoria, BC, Canada, 2016.

21. Khan, Z.H.; Gulliver, T.A. A macroscopic traffic model for traffic flow harmonization. Eur. Transp. Res. Rev. 2018, 10. [CrossRef]

22. Mohan, R.; Ramadurai, G. State-of-the art of macroscopic traffic flow modelling. Int. J. Adv. Eng. Sci. Appl. Math. 2013, 5, 158-176. [CrossRef]

23. Zhang, H.M.; Jin, W.L. Kinematic Wave Traffic Flow Model for Mixed Traffic. Transp. Res. Rec. 2002, 1802, 197-204. [CrossRef]

24. Ngoduy, D. Multiclass first-order traffic model using stochastic fundamental diagrams. Transportmetrica 2011, 7, 111-125. [CrossRef]

25. Gupta, A.K.; Dhiman, I. Analyses of a continuum traffic flow model for a nonlane-based system. Int. J. Mod. Phys. C 2014, 25, 1-24. [CrossRef]

26. Mohan, R.; Ramadurai, G. Heterogeneous traffic flow modelling using second-order macroscopic continuum model. Phys. Lett. Sect. A Gen. At. Solid State Phys. 2017, 381, 115-123. [CrossRef]

27. Prigogine, I.; Herman, R. Kinetic Theory of Vehicular Traffic; Elsevier: New York, NY, USA, 1971.

28. Paveri-Fontana, S.L. On Boltzmann-like treatments for traffic flow: A critical review of the basic model and an alternate proposal for dilute traffic analysis. Transp. Res. 1975, 9, 225-235. [CrossRef]

29. Phillips, W.F. A kinetic model for traffic flow with continuum implications. Transp. Plan. Technol. 1979, 5, 131-138. [CrossRef]

30. Roe, P.L. Approximate Riemann Solvers, Parameter Vectors, and Difference Schemes. J. Comput. Phys. 1997, 135, $250-258$. [CrossRef]

31. Imran, W.; Khan, Z.H.; Aaron Gulliver, T.; Khattak, K.S.; Nasir, H. A macroscopic traffic model for heterogeneous flow. Chin. J. Phys. 2020, 63, 419-435. [CrossRef]

32. Mohazzabi, P.; Cumaranatunge, L. Graham's law of effusion in dense systems with nonuniform interactions. Can. J. Phys. 2003, 81, 1121-1129. [CrossRef]

33. Lesort, J.; Bourrel, E.; Henn, V. Various Scales for Traffic Flow Representation: Some Reflections. In Traffic and Granular Flow '03; Hoogendoorn, S.P., Luding, S., Bovy, P.H.L., Schreckenberg, M., Wolf, D.E., Eds.; Springer: Berlin/Heidelberg, Germany, 2005; ISBN 978-3-540-28091-0.

34. Kachroo, P.; Al-nasur, S.; Wadoo, S.; Shende, A. Pedestrian Dynamics; Springer: Berlin/Heidelberg, Germany, 2008; Volume 53, ISBN 9788578110796.

35. Harten, A.; Hyman, J.M. Self adjusting grid methods for one-dimensional hyperbolic conservation laws. J. Comput. Phys. 1983, 50, 235-269. [CrossRef]

36. De Moura, C.A.; Kubrusly, C.S. The Courant-Friedrichs-Lewy (CFL) Condition: 80 Years After Its Discovery; Springer: Berlin, Germany, 2013.

37. Greenshields, B.D.; Bibbins, J.R.; Channing, W.S. A study of traffic capacity. Highw. Res. Board. 1935, 14, 458. 